\title{
Multiple Embolic Strokes Revealing a Huge Mitral Annular Calcification
}

Lamiae Khelifi, MD*, Sara Hassani, MD, Ilyass Asfalou, MD, Aatif Benyass, MD

Department of Cardiology, Military hospital Mohamed V of Rabat, Morocco

DOI: $\underline{10.36347 / \text { sjmcr.2020.v08i05.024 }}$

| Received: 09.05.2020 | Accepted: 16.05.2020 | Published: 27.05.2020

*Corresponding author: Lamiae Khelifi

Abstract

Background: Mitral annular calcification (MAC) is a degenerative process which commonly occurs in the elderly. Patients with MAC have a higher prevalence of left atrial enlargement, left ventricular enlargement, atrial fibrillation, conduction defects, mitral regurgitation, and mitral stenosis. In addition, these patients have a higher incidence of cardiovascular events, thromboembolic events. Case Presentation: A 75-year-old Moroccan woman with no cardiovascular risk factors presented a transitory ischemic stroke. A transthoracic echocardiogram revealed a mitral annular mass $(21 \times 12 \mathrm{~mm})$ attached to the auricular side of the posterior mitral valve leaflet. The cerebral CT scan showed lesions of a previous embolic stroke. The cardiac CT-Scan and a cardiac MRI confirmed the diagnosis of a mitral annular calcification. Conclusions: In the present case, the mitral annular calcification did not require any treatment. Instead, patients are managed according to the presence of heart block, mitral regurgitation and other cardiovascular signs and symptoms.

Keywords: Mitral annular calcification, stroke.

Copyright @ 2020: This is an open-access article distributed under the terms of the Creative Commons Attribution license which permits unrestricted use, distribution, and reproduction in any medium for non-commercial use (NonCommercial, or CC-BY-NC) provided the original author and source are credited.

\section{INTRODUCTION}

Approximately one-fifth of all ischemic strokes are cardioembolic. Mitral annular calcification (MAC) is a progressive, degenerative condition with a prevalence that increases proportionately with age $[2$, $3]$. It is a result of progressive calcification of the fibrous mitral annulus [4], most commonly involving the posterior portion. It is usually confined to the annulus and leaflet base, occasionally it can cause a leaflet restriction. In less than $1 \%$ of MAC cases, the calcified mass may contain a radiolucent core, a finding that has been termed "caseous" calcification of the mitral annulus [1, 7]. Therefore, the term mitral annular calcification with central softening (MACCS) is a more apt description without the errant connotation [6]. Complete surgical resection is the treatment of choice for reducing the symptoms and preventing embolization, as well as for pathological diagnosis [8].

\section{Case Presentation}

A 75-year-old Moroccan woman with no history of heart disease complained of transitory hemiplegia and was referred to our hospital. She had been taking care of in the neurovascular unit and was referred to our unit to purchase the investigations. She had no history of thromboembolism or endocarditis.
She had no significant social and environmental history. Her medication list included lansoprazole. She had no family history of cardiovascular diseases.

She was afebrile, with a blood pressure of $125 / 70 \mathrm{mmHg}$ and a regular pulse rate of 70 beats per minute. Her oxygen saturation was $96 \%$ on ambient air. There was no evidence of lung crakles, cardiac murmur, and abdominal tenderness.

Her neurological examinations showed no sign of deficit, she completely recovered. Blood investigations showed: hemoglobin $11.0 \mathrm{mg} / \mathrm{dl}$, white blood cells $6000 / \mathrm{mm} 3$, and platelets $176,000 / \mathrm{mm} 3$. Her liver and renal function tests revealed aspartate aminotransferase $34 \mathrm{IU} / \mathrm{l}$, alanine aminotransferase 26 $\mathrm{IU} / \mathrm{l}$, and serum creatinine $13 \mathrm{mg} / \mathrm{dL}$. Her C-reactive protein was $0.16 \mathrm{mg} / \mathrm{dl}$.

Her serum electrolyte tests were within normal limits, including calcium and inorganic phosphorus. In addition, her brain natriuretic peptide was raised at $605.1 \mathrm{pg} / \mathrm{dl}$. Her blood cultures were negative.

A chest X-ray showed no cardiomegaly and no pulmonary congestion. An electrocardiogram suggested left ventricular hypertrophy with no STT wave changes. 
A transthoracic echocardiography revealed a mitral annular mass $(21 \times 12 \mathrm{~mm})$ attached to the auricular side of the posterior mitral valve leaflet (Fig $1 \& 2$ ). The echogenicity of the mass was similar to MAC. The mitral valve was mildly thickened with no mitral stenosis. Left ventricular systolic function was normal. Cardiac computed tomography demonstrated a calcified cardiac mass in the mitral annulus with heavy MAC (Fig-3). The cardiac MRI confirmed the diagnosis of a mitral annular calcification (Fig-4).

As the mass was not mobile, and did not block the mitral leaflets we decided not to undergo surgical resection of the mass and to establish a medical monitoring. We let the patient under aspirin and lansoprazole.

\section{DisCUSSION}

The origin of MAC is unclear, approximately $20 \%$ of patients suggesting a potential role of phosphocalcic metabolism abnormalities [4]. In addition, hypercoagulable state was reported to be a risk factor of MAC [5]. MAC is defined by pathological findings of calcified nodules in an amorphous background of fibrin, with degeneration and focal inflammation. It is often difficult to distinguish MAC from other cardiac tumors, vegetation, and thrombus when using single imaging modalities. Caseous calcification of the mitral annulus (CCMA) is a rare variant of MAC, which is observed in $0.067 \%$ of all echocardiographic exams, and is present in $0.63 \%$ of all patients with MAC [9]. The typical appearance of CCMA is an echo-dense mass containing central areas of echolucencies at the posterior periannular region of the mitral valve on echocardiography [10]. The prevalence of cardioembolic events associated with CCMA is $19.2 \%$ which is higher than the CVE reported in MAC (11.8\%) [1]. In addition, improvements in echocardiography and use of cardiac imaging modalities during the last decade have helped to uncover this rare variety of MAC. CCMA was directly linked to cerebral embolization $[11,12]$. The possible mechanisms for cerebral embolization from CCMA lesions include:

1. Spontaneous fistulization and embolization of caseous material that may leak directly into the left atrium or ventricle [11, 13];

2. Embolization of caseous necrotic debris from the CCMA lesion;

3. Embolization of calcium and cholesterol particles.

In the case of our patient, it seems that embolization of calcium and cholesterol particles is the most plausible thesis since there was no evidence of caseous necrosis.

MAC undergo surgical resection due to the high risk of embolization, which remains the diagnostic and therapeutic standard. The sizes of MAC were recently reported to range from several millimeters to $90 \mathrm{~mm}$ [3]. Patients with MAC are mostly asymptomatic at presentation, although the masses can cause symptoms related to obstruction or embolization, such as dyspnea and syncope [3]. Furthermore, MAC with a highly mobile characteristic is at high risk of stroke or other systemic embolism. However, postoperative recurrence due to incomplete resection has been reported [5]. Thus, careful clinical and echocardiographic follow-up is required after surgery.

\section{CONCLUSION}

We report a case of $\mathrm{MAC}$ revealed by a transitory stroke. MAC is considered as passive, degenerative process but it can also be a result of an active atherosclerotic process. Caseous calcification of the mitral valve should be considered in the differential diagnosis of cardiac tumors. Such calcification usually carries a benign prognosis, but patients so diagnosed require careful follow-up observation if they develop new symptoms or have a high propensity for tissue calcium deposition (as in chronic renal failure).

\section{List of Abbreviations}

MAC: Mitral annular calcification

CCMA: Caseous calcification of the mitral annulus

CVE: Cardiovascular events

Disclosures: The authors declare no conflict of interest.

\section{ACKNOWLEDGEMENTS}

I would like to express my special thanks of gratitude to my teachers, who gave me the golden opportunity to do this wonderful project. Secondly i would also like to thank my parents and friends who helped me a lot in finalizing this article.

\section{REFERENCES}

1. Dietl CA, Hawthorn CM, Raizada V. Risk of cerebral embolization with caseous calcification of the mitral annulus. The open cardiovascular medicine journal. 2016;10:221.

2. Harpaz D, Auerbach I, Vered Z, Motro M, Tobar A, Rosenblatt S. Caseous calcifcation of the mitral annulus: a neglected, unrecognized diagnosis. J Am Soc Echocardiogr, 2001; 14:825-831.

3. Payvandi LA, Rigolin VH. Calcifc Mitral Stenosis. Cardiol Clin, 2013; 31:193-202.

4. Eleid MF, Foley TA, Said SM, Pislaru SV, Rihal CS. Severe Mitral Annular Calcifcation: Multimodality Imaging for Therapeutic Strategies and Interventions. JACC Cardiovasc Imaging, 2016; 9:1318-1337.

5. Barasch E, Gottdiener JS, Larsen EK, Chaves PH, Newman AB, Manolio TA. Clinical signifcance of calcifcation of the fbrous skeleton of the heart and aortosclerosis in community dwelling elderly. The 
Cardiovascular Health Study (CHS). Am Heart Journal, 2006; 151:39-47

6. Lyle MA, Snipelisky DF, Aggarwal NR, Miller FA, Anavekar NS. Exuberant mitral annular calcification. The international journal of cardiovascular imaging. 2017 May 1;33(5):61521.

7. Deluca G, Correale M, Ieva R, Del Salvatore B, Gramenzi S, Di Biase M. The incidence and clinical course of caseous calcifcation of the mitral annulus: a prospective echocardiographic study. Journal Am Soc Echocardiogr, 2008; 21:828-833.

8. Nakamaru R, Oe H, Iwakura K, Masai T, Fujii K. Calcified amorphous tumor of the heart with mitral annular calcification: a case report. Journal of medical case reports. 2017 Dec 1;11(1):195.

9. Harpaz D, Auerbach I, Vered Z, Motro M, Tobar A, Rosenblatt S. Caseous calcification of the mitral annulus: a neglected, unrecognized diagnosis. Journal Am Soc Echocardiogr, 2001;14(8): 825-31.
10. Deluca G, Correale M, Ieva R, Del Salvatore B, Gramenzi S, Di Biase $M$. The incidence and clinical course of caseous calcification of the mitral annulus: a prospective echocardiographic study. J Am Soc Echocardiogr, 2008; 21(7): 82833.

11. Higashi H, Ohara T, Nakatani S, Hashimoto S, Torii T, Miyashita K, Naritomi H, Kitakaze M. A case of caseous calcification of the mitral annulus: a potential source of embolic stroke. Journal of cardiology cases. 2010 Dec 1;2(3):e141-3.

12. Sequeira A, Morris L, Patel B, Duvall L, Gali D, Menendez D, Alexander G. Calcific mitral stenosis in the hemodialysis patient. Hemodialysis International. $2014 \mathrm{Jan} ; 18(1): 212-4$.

13. Chevalier B, Reant P, Laffite S, Barandon L. Spontaneous fistulization of a caseous calcification of the mitral annulus: an exceptional cause of stroke. Eur J Cardiothorac Surg, 2011; 39(6):e1845 . 